\title{
$\angle$ Research Square \\ The Impact of COVID-19 pandemic Lockdown on the utilization of acute surgical care, retrospective, multicenter analysis
}

Jaroslav Presl

Paracelsus Medical University https://orcid.org/0000-0002-2899-6706

Martin Varga

Paracelsus Medical University Salzburg: Paracelsus Medizinische Privatuniversitat

Christof Mittermaier

Order of Merciful Brothers Salzburg Hospital: Krankenhaus der Barmherzigen Bruder Salzburg

Stefan Mitterwallner

Hospital Oberndorf, Teaching Hospital of the Paracelsus Medical University Salzburg

Michael Weitzendorfer

Paracelsus Medical University Salzburg: Paracelsus Medizinische Privatuniversitat

\section{Kurosch Borhanian}

Paracelsus Medical University Salzburg: Paracelsus Medizinische Privatuniversitat

\section{Andreas Heuberger}

Hospital Oberndorf, Teaching Hospital of the Paracelsus Medical University Salzburg

\section{Helmut Weiss}

Order of Merciful Brothers Salzburg Hospital: Krankenhaus der Barmherzigen Bruder Salzburg

\section{Klaus Emmanuel}

Paracelsus Medical University Salzburg: Paracelsus Medizinische Privatuniversitat

\section{Burkhard von Rahden}

Paracelsus Medical University Salzburg: Paracelsus Medizinische Privatuniversitat

Oliver Owen Koch ( $\nabla$ o.koch@salk.at )

Paracelsus Medical University Salzburg: Paracelsus Medizinische Privatuniversitat

\section{Research article}

Keywords: COVID-19, Pandemic, Emergency, Appendectomy, Colorectal surgery, Inpatient, General surgery, Lockdown

Posted Date: September 2nd, 2020

DOI: https://doi.org/10.21203/rs.3.rs-70079/v1 
License: (c) (i) This work is licensed under a Creative Commons Attribution 4.0 International License. Read Full License 


\section{Abstract}

\section{Background:}

The emergency general surgery encompasses the care of critically ill patients, with a potentially high mortality if delayed. As some medical disciplines have reported a strong decrease of emergencies during the COVID-19 pandemic, remains the effect of the Lockdown on the general surgery emergencies unclear.

\section{Methods:}

This study is a retrospective, multicentre analysis of the general surgery emergency operations performed during the 2020 Lockdown and in the same period of 2019 in three centers covering the surgical care of the area Salzburg-North, Austria.

\section{Results:}

In total 165 emergency surgeries were performed in the study period of 2020 compared to 287 in Year 2019. This is a significant decrease of 122 (42.5\%) emergency surgeries during the COVID-19 Lockdown $(p=0.005)$. The average length of hospital stay in the 2019 was in median 4 days and was reduced to 3 days during the Lockdown. Appendectomy remained the most performed emergency surgery for the both periods but the operations count reduced to less than a half with 72 cases in 2019 and 33 cases in 2020 $(p=0.118)$. Considering the ration of appendectomy vs all emergency surgeries, it represented $25 \%$ in 2019 and $20 \%$ in 2020 .

The emergency colon surgery observed the strongest decrease of $75 \%$ from 17 cases in 2019 to 4 in 2020. In addition, the emergency abdominal wall hernia, cholecystectomies for acute cholecystitis, small surgeries and proctological emergencies recorded drops of $70 \%, 39 \%, 33 \%$ and $47 \%$ respectively.

A strongest reduction in frequency of 6 of 13 main categories of emergency surgeries was reported from Center 1, which was the only COVID designed Center ("Hot" hospital) in the examined region.

\section{Conclusions:}

The emergency general surgery is an essential service that continues to run under any circumstances. Our data showed that the COVID-19 related restriction and the fear of being infected with COVID-19 in the hospital result in a significant decrease of the utilization of acute surgical care. Policies and modern alternatives are needed to ensure continued access to specialized services to prevent patients from harm.

\section{Introduction:}

The emergency general surgery encompasses the care of critically ill surgical patients. Fast diagnosis and accurate therapy of an abdominal emergency can prevent a high lethality of potential intra- 
abdominal infection, which can reach up to $40 \%$ in case of abdominal sepsis [1-4].

As the first cases of a new viral pneumonia, today known as COVID-19 caused by corona virus SARSCoV2, were reported from Wuhan/China in December 2019, nobody knew that in March 2020 the COVID19 would become pandemic [5]. The high mortality of the disease, which initially reached $7.2 \%$ in Italy [6], forced the authorities, by missing causal therapy or vaccination, to a strict exposition prophylaxis ("Lockdown") to slow down the spread. The first COVID-19 positive case in the state Salzburg, Austria was documented on 29th February 2020. Because of the growing caseload of COVID-19 positive patients, the government decided about the first steps to restrict public life on 16th March 2020. These measures were gradually tightened until 14th April 2020 when parts of the restrictions withdrawn. In order to avoid the spread in the public hospitals and to save health care capacities, all elective interventions (incl. operations) were postponed and the population was encouraged to avoid the hospitals except emergency cases. Simultaneously with the increasing numbers of infected patients and growing fear of possible overwhelming number of COVID-19 patients, another effect of the Lockdown was reported from abroad. In the USA, the nine High-Volume catheterization laboratories reported a decrease activation for STelevation myocardial infarction (STEMI) of 38\% [7]. The similar trend could be observed by neurologists from China and USA, who reported a dramatic decline of acute strokes of $30-50 \%[8,9]$.

At this moment, there is no knowledge about the effect of the Lockdown on the presentation of the general surgery emergencies, which could be crucial to prevent patients from severe adverse effects. The aim of this work is to investigate whether there have been any changes in the surgical emergency operations in the north of the state Salzburg, Austria (supply region 51) during the national Lockdown.

\section{Methods:}

We have conducted a retrospective analysis of all general surgery emergency surgeries performed in the entire emergency Region 51 in the period from 1 March to the 15th of April 2020 (= 6 weeks). The Region 51 is a defined north part of the state Salzburg, Austria with 364.797 inhabitants, which is $66 \%$ of the population of the whole state Salzburg. There are three general surgery centers providing emergency surgical care in this region:

Center 1: The Department of Surgery of the Paracelsus Medical University Salzburg. This center also includes the data of its external Surgical Division located in the city Hallein, Austria. (PMU + Hallein)

Center 2: The Hospital of the Brothers of St. John of God in Salzburg (BHB)

Center 3: Department of Surgery, Hospital Oberndorf, Teaching Hospital of the Paracelsus Medical University of Salzburg, Oberndorf, Austria. (Oberndorf)

All three centers submitted their data during the study period. Demographic data (age, sex), the length of hospital stay (LOS), the type of acute surgery, the indication for surgery and the mortality rates were analyzed. For comparison, the equal parameters from the three centers in the same time period $1 \mathrm{March}$ 
to the 15th of April of the year 2019 were used. The surgeries were divided into 13 main categories, defined upon the most frequent clinical diagnosis, according to which the statistic comparation followed. (appendectomy, cholecystectomy, bowel ischemia, small bowel perforation, acute abdominal wall hernia, bowel obstruction, small acute surgeries = small one day surgeries, colon emergencies, proctological emergencies, revision operations after any index operation, emergency thorax surgery, abdominal trauma surgeries, stomach bleeding or perforation surgeries) Table 1. Only Center 1 was dedicated as a regional COVID Center ("Hot" hospital) so it covered the care of COVID and Non-COVID patients. This took place in a dedicated COVID Clinic located on the Campus of the University Hospital in Salzburg [10]. Center 2 and 3 remained COVID free during the Lockdown. All patients included in the study were considered COVID-19 negative.

\section{Statistical Analysis:}

Statistical analysis was performed using SPSS-Statistical-Analysis Software (SPSS Inc., Chicago, IL, USA). All data were tested for normal distribution by Kolmogorow-Smirnow-Test. Comparison data was done using paired-t-test respectively Wilcoxon signed rank test on a per subject basis. Population homogeneity was conducted using independent t-Test or Mann-Whitney-U-Test. If normally distributed, measurements were additionally presented as means and standard deviation (SD). $p<0.05$ was regarded as statistically significant.

\section{Results:}

In the region Salzburg North, 165 emergency surgeries (ES) were performed in the study period of 2020 compared to 287 ES in 2019. This is a significant decrease of $122(42.5 \%)$ emergency surgeries during the COVID-19 Lockdown ( $p=0.005$ ) (Table 1). In total 248 (54.9\%) male and 204 (45.1\%) female patients underwent an emergency operation in 2019 and 2020. The distribution according to the gender remained equal in 2019 (144 male vs. 143 female). During the COVID-19 Lockdown in 2020, 104 male patients $(63 \%)$ were operated compared to 61 female patients (37\%). The median age of the operated patients in 2019 vs 2020 was 52 years $( \pm 20.3)$ and 52 years $( \pm 20.1)$ without showing any significant difference $(p=$ 0.991). Comparing the Length of hospital stay (LOS) between the year 2019 and 2020 in every single centre, we have not found any significant difference, but the average LOS in the 2020 was reduced from 4 to 3 days in 2020 in comparison to the year before (Table 2). The appendectomy remains the most frequent performed emergency surgery for both time periods, but the operations count reduced from 72 cases to less than a half, 33 cases in the year $2020(p=0.118)$. Considering the ration of appendectomy vs all emergency surgeries, it represented $25 \%$ in 2019 and $20 \%$ in 2020 . The statistic significant decrease was reported only in the Centre 1 ("Hot" hospital) from 51 Appendectomies in 2019 to 14 in 2020 ( $p=$ 0.047). The second most frequent surgeries were the small surgeries (including surgery for small skin inflammation - but not the proctology), showing decrease of $20 \%(p=0.156)$ with 59 procedures in 2019 and 40 in 2020. The emergency colon surgery observed the strongest decrease of $75 \%$ from 17 cases in 2019 to 4 in 2020. And it was again the Centre 1 reporting the fall from 11 cases in 2019 to only 1 case in 2020 (Table 1). The amount of cholecystectomies for acute cholecystitis dropped from 33 operations to 
20 during the Lockdown $(p=0.262)$ and again the Centre 1 counted the drop of more than $62 \%$. In general, the Centre 1 recorded the significant drops in 6 of the defined main categories of the surgeries (Table 1)(Fig. 1).

\section{Discussion:}

The COVID-19 pandemic Lockdown caused a significant reduction of the general surgical emergency operations of $42.5 \%(p=0.005)$ in the entire Region 51 . This noteworthy reduction of emergencies was reported also from other medical specialties around the globe. Garcia et al. reported about diminished activations of US cardiac catheterization laboratory for STEMI in the COVID-19 era [7]. The Medscape Medical News also announce a $30 \%$ decreased rates of major stroke in Chicago [8]. Furthermore, the preliminary data to Stroke care from Shanghai reported a decrease of thrombectomies by $50 \%$ in the first month after the Spring Festival (24th to 30th January 2020) [9]. Those specialties discussed the reduction of stress and the deceleration of public live as a potential reason for such a decline of cardiovascular emergencies [7-9].

As the role of negative stress in functional gastrointestinal disorders is well known, the effect of stress on development of abdominal emergencies has not been described so far [11].

The strong decrease of acute appendectomies and acute cholecystectomies of $54 \%$ and $39 \%$ respectively in the year 2020 in our cohort is striking. Many different deviations of stated therapies, to minimize the risk of COVID-19 cross infection are being reported. Patel et al. described shortly ago an increased consumption of a conservative antibiotic therapy for uncomplicated appendicitis, during the Lockdown in England [12]. This therapy is in the literature stated as a feasible and save option, but with an increased risk for appendicitis recurrence $[13,14]$. Patel also reported a deviation from the guidelines and an exclusively use of a conservative therapy as a first line treatment for acute cholecystitis $[12,15]$.

Based on this knowledge, all centers in Region 51 confirmed no change to the state of the art operative therapy of acute appendicitis and cholecystitis.

The official recommendation of the authorities to the public was to avoid hospitals until a "real" emergency occurs. Furthermore, the patients were encourage to discuss the general practitioners in the first line. According to that, the general practitioners haven't reported any increase of patients load or change of the therapeutic pathways, including the reporting of all patients suspected of abdominal emergency to a surgical specialist. If the reduction of the case load of emergencies or preferred antibiotic treatment or both are responsible for reduction of acute surgeries in our region stays unknown.

The strict politics of the authorities, with the clear aim of preparing the medical system for a huge number of COVID-19 positive patients, as seen in Italy, caused a dramatic reduction of all elective surgeries of $46 \%$ during the Lockdown. We have also observed a faster relocation of the Non-COVID patient's therapy to the outpatient setting, leading to a shorter median LOS of 3 days (reduction of $25 \%$ ) in the examined 
period of 2020. The same trend was reported by Patel et al. in his cohort with a significant reduction of LOS from 3 to 2 days $(p=0.04)$ [12].

The general avoidance of medical care due to the social distancing or due to the concerns of patients of being infected with COVID-19 in the hospital, remain the most common reasons for the decrease of emergency surgeries in entire Region 51. To underline these facts, we have identified a noticeable decrease in the frequency of 6 of 13 main categories of emergency surgeries in Center 1 , which was the only COVID designed Center ("Hot" hospital) in the examined Region 51. Huang et al. already reported this potential perception of personal danger, dramatically reducing the willingness of patients to visit an emergency department of the "Hot" hospital, in 2003, during the last epidemic of SARS from Taipei, Taiwan [16]. The authors reported a reduction of up to $51.6 \%$ in daily visits of emergency departments at the peak of the SARS epidemic, which persisted for 3 months after the end of the epidemic. The longlasting effect of people's fear of designed SARS centers was reported from the same hospital in Taipei and published in 2008. The results show, that in long-term observation the outpatient department of the general surgery department had still significantly less patients treated at 4 years after SARS epidemic [17].

The further consequences of the COVID-19 pandemic for acute surgical patients, who did not received an appropriate, guidelines conform, therapy remain still unclear and should be further examined. We cannot forget the potentially high mortality of severe intra-abdominal infections, which could reach up to $40 \%$ in case of sepsis [3]. The Statistic Austria reported recently the mortality data for Austria during Lockdown (Calendar Week 12 to 16), in comparison to the average for the period in the years 2016-2019. They found a significant growth of the week mortality in the population of 65 Years and older. The highest growth of 252 deaths (16.9\%) in the calendar week 12 and growth of 200 deaths (14.9\%) in the week 14 was registered again in the group of 65 Years and older. The growth of mortality in State Salzburg was $4 \%$ in calendar week 14 and remained low during the whole Lockdown, without a significant change to previous years. Unfortunately, it is not possible to identify the reasons for reported deaths. The increased mortality confirms the frailty of elderly people and reflects the results of studies taking the emergency abdominal surgery in the elderly under the scope. These are showing a higher mortality of $8-16 \%$ in this group even being treated [18-20]. Just to reflect the COVID Situation during our examined period, the total number of COVID positive deaths in Austria was 384 and 27 in whole State Salzburg, Austria [21, 22].

\section{Conclusions:}

The emergency general surgery is an essential health service that continues to run under any circumstances. Our data showed that the COVID-19 related restrictions lead to a significant decrease of the utilization of acute surgical care. The increased overall mortality of people being 65 years or older, must be a caution for public health care providers, that should encourage people to continue the use of public health care services, especially when suffering from serious medical complains. New policies are 
needed to enable a save access to specialized health services for patients twenty-four seven, especially in the situations like caused by COVID-19.

\section{Abbreviations}

STEMI -ST-elevation myocardial infarction

PMU+Hallein -The Department of Surgery of the Paracelsus Medical University Salzburg, this Center includes also the data of its external Surgical Division located in the City Hallein

BHB -The Hospital of the Brothers of St. John of God in Salzburg

Oberndorf -Department of Surgery, Hospital Oberndorf, Teaching Hospital of the Paracelsus Medical University of Salzburg, Oberndorf, Austria.

ES -Emergency surgeries

LOS -Length of stay

Region 51 -Salzburg North, Austria

Elderly - 65 Years and older

\section{Declarations}

\section{Ethics approval and consent to participate}

- Not applicable

\section{Consent for publication}

- Not applicable

\section{Availability of data and materials}

- All data generated or analyzed during this study are included in this published article [and its supplementary information files]

\section{Competing interests}

- The authors declare that they have no competing interests

\section{Funding}

- There was no funding for this work 


\section{Authors' contributions}

- PJ Data collection (PMU + Hallein) and writing the manuscript as a main author

- VM Data collection (PMU + Hallein) and major corrections of the manuscript

- MCh Data collection (BHB)

- MS Data collection (Oberndorf)

- WM Data collection (PMU + Hallein) and major corrections of the manuscript

- BK Data collection (PMU + Hallein) and language corrections of the manuscript

- HA Data collection (Oberndorf)

- WH Data collection (BHB)

- EK Scientific supervision of the manuscript

- BvR Preparing the Figures and Tables and language correction of the manuscript

- KO Scientific supervision of the manuscript, major correction of the manuscript, corresponding author

\section{Acknowledgements}

We thank Ms. Stefanie Frank, LLB.oec. for her excellent work analyzing case numbers, preparing graphics and statistics for the publication.

\section{References}

1. Scott J, Olubode A, Brat G, Rose J, Zogg Ch, Haider A, et al. Use of National Burden to Define Operative Emergency General Surgery. Jama Surg. 2016;151(6):e160480.

DOI:10.1001/jamasurg.2016.0480.

2. Markar S, Vidal-Diez A, Patel K, Maynand W, Tikanova K, Murray A, et al. Comparison of Surgical Intervention and Mortality for Seven Surgical Emergencies in England and the United States. Ann Surg. 2019;270:806-12. DOI:10.1097/SLA.0000000000003518.

3. Mačiulienè A, Maleckas A, Krisciukaitis A, Maciulis V, Vencius J, Macas A, et al. Predictors of 30-Day In-Hospital Mortality in Patients Undergoing Urgent Abdominal Surgery Due to Acute Peritonitis Complicated with Sepsis. Med Sci Monit. 2019;25:6331-40. DOI:10.12659/MSM.915435.

4. Sartelli M, Catena F, Ansaloni L, Leppaniemi A, Taviloglu K, van Goor H: Complicated intra-abdominal infections in Europe: a comprehensive review of the CIAO study. World J Emerg Surg 2012, 7:36

5. Zhu N, Zhang D, Wang W, Xingwang L, et al. A Novel Coronavirus from Patients with Pneumonia in China, 2019. N Engl J Med. 2020;382:727-33. DOI:10.1056/NEJMoa2001017.

6. Onder G, Rezza G, Brusaferro S. Case-Fatality Rate and Characteristics of Patients Dying in Relation to COVID-19 in Italy. JAMA. 2020;323(18):1775-6. DOI:10.1001/jama.2020.4683. 
7. Garcia S, Albaghdadi M, Meraj P, Schmidt Ch, Garberich R, Jaffer F, et al. Reduction in ST-Segment Elevation Cardiac Catheterization Laboratory Activations in the United States during COVID-19 Pandemic. J Am Coll Cardiol 2020 Jun 9;75(22):2871-2872. DOI: 10.1016/j.jacc.2020.04.011.

8. McNamara D. COVID-19: Are Acute Stroke Patients Avoiding Emergency Care: https://www.medscape.com/viewarticle/928337 (Accessed: 19 Aug 2020).

9. Zhao J, Rudd A, Liu R. Challenges and Potential Solutions of Stroke Care During the Coronavirus Disease 2019 (COVID-19) Outbreak. Stroke. 2020 May;51(5):1356-7. DOI:10.1161/STROKEAHA.120.029701.

10. Presl J, Weitzendorfer M, Varga M, Borhanian K, Ciftci S, Emmanuel K, et al. Surgical strategies during the COVID-19 crisis: The Salzburg concept. Am J Surg May 12, 2020 DOI:https://doi.org/10.1016/j.amjsurg.2020.05.006.

11. Mönnikes H, Tebbe J, Hildebrandt M, Arck P, Osmanoglou E, Rose M, et al. Role of Stress in functional gastrointestinal disorders. Dig Dis. 2001;19(3):201-11. DOI:10.1159/000050681.

12. Patel R, Hainsworth AJ, Patel Devlin K, Karim JH. A. Frequency and severity of general surgical emergencies during the COVID-19 pandemic: single-centre experience from a large metropolitan teaching hospital. Ann R Coll Surg Engl 2020 Jun 27; 102(6):1-6. DOI: 10.1308/rcsann.2020.0147. Online ahead of print.

13. $10.1001 /$ jama.2015.6154

Salminen P, Paajanen H, Rautio T, Nordström P, Aarnio M, Rantanen T, et al. Antibiotic therapy vs appendectomy for treatment of uncomplicated acute appendicitis: The APPAC randomized clinical trial. JAMA 2015 Jun 16;313(23):2340-8. DOI: 10.1001/jama.2015.6154.

14. Severio S, Podda M, Simone B, Ceresoli M, Augustin G, Gori A. Diagnosis and treatment of acute appendicitis: 2020 update of WSES Jerusalem guidelines. World J Emerg Surg. 2020;15:27.

15. Ansaloni L, Pisano M, Coccolini F, Peitzmann AB, Fingerhut A, Catena F. 2016 WSES guidelines on acute calculous cholecystitis. World J Emerg Surg. 2016;11:25.

16. Huang H, Yen D, Kao W, et al. Declining Emergency Departemnt Visits and Costs During the Severe Acute Respiratory Syndrome (SARS) Outbreak. J Formos Med Assoc. 2006 Jan;105(1):31-7. DOI:10.1016/S0929-6646(09)60106-6.

17. Chu D, Chen RC, Ku CY, Chou P. The impact of SARS on hospital performance. BMC Health Serv Res. 2008 Nov 6;8:228. DOI: 10.1186/1472-6963-8-228.

18. Kraemer M, Franke C, Ohmann C, Yang Q, Group, Acute Abdominal Pain Study. Acute appendicitis in late adulthood: incidence, presentation, and outcome. Results of a prospective multicenter acute abdominal pain study and a review of the literature. Langenbecks Arch Surg. 2000 Nov;385(7):47081.

19. Wiggins T, Markar S, Mackenzie H, Jamel S, Askari A. Faiz O Evolution in the management of acute cholecystitis in the elderly: population-based cohort study. Surg Endosc. 2018;32(10):4078-86.

20. Fukuda N, Wada J, Niki M, Sugiyama Y, Mushiake H. Factors predicting mortality in emergency abdominal surgery in the elderly. World J Emerg Surg. 2012;7:12. 
21. Statistik Austria. https://www.statistik.at/web_de/presse/122934.html (Accessed: 16.6.2020). 22. Coronavirus disease 2019 (COVID-19)Situation Report 86: https://www.who.int/docs/defaultsource/coronaviruse/situation-reports/20200415-sitrep-86-covid-19.pdf?sfvrsn=c615ea20_6 (Accessed: 4.8.2020).

\section{Tables}




\begin{tabular}{|c|c|c|c|c|}
\hline & & $\begin{array}{l}\text { Period } \\
\text { 1.3.-15.4.2019 }\end{array}$ & $\begin{array}{l}\text { Period } \\
1.3 .-15.4 .2020\end{array}$ & \\
\hline Emergency operations & Total & 287 & 165 & $p=0.005$ \\
\hline Appendectomy & $\mathrm{n}=105$ & 72 & 33 & $p=0.118$ \\
\hline PMU+Hallein & & 51 & 14 & $p=0.047$ \\
\hline $\mathrm{BHB}$ & & 14 & 15 & $p=0.970$ \\
\hline Hospital Oberndorf & & 7 & 4 & $p=0.438$ \\
\hline Cholecystectomy & $\mathrm{n}=54$ & 33 & 20 & $p=0.262$ \\
\hline PMU+Hallein & & 23 & 10 & \\
\hline $\mathrm{BHB}$ & & 7 & 6 & \\
\hline Hospital Oberndorf & & 4 & 4 & \\
\hline Bowel ischemia & $n=4$ & & & \\
\hline PMU+Hallein & & 3 & 1 & \\
\hline Small bowel perforation & $n=6$ & 2 & 4 & \\
\hline PMU+Hallein & & 2 & 2 & \\
\hline $\mathrm{BHB}$ & & 0 & 1 & \\
\hline Hospital Oberndorf & & 0 & 1 & \\
\hline $\begin{array}{l}\text { Emergency abdominal wall } \\
\text { hernia }\end{array}$ & $\mathrm{n}=17$ & 13 & 4 & $P=0.425$ \\
\hline PMU+Hallein & & 4 & 2 & \\
\hline $\mathrm{BHB}$ & & 5 & 2 & \\
\hline Hospital Oberndorf & & 4 & 0 & \\
\hline Bowel obstruction & $n=33$ & 15 & 18 & $p=0.324$ \\
\hline PMU+Hallein & & 9 & 10 & \\
\hline $\mathrm{BHB}$ & & 6 & 6 & \\
\hline Hospital Oberndorf & & 0 & 2 & \\
\hline Small acute surgeries & $\mathrm{n}=99$ & 59 & 40 & $p=0.156$ \\
\hline PMU+Hallein & & 42 & 25 & $p=0.225$ \\
\hline $\mathrm{BHB}$ & & 6 & 5 & \\
\hline Hospital Oberndorf & & 11 & 10 & \\
\hline
\end{tabular}




\begin{tabular}{|c|c|c|c|c|}
\hline Colon emergencies & $\mathrm{n}=21$ & 17 & 4 & $p=0.421$ \\
\hline PMU+Hallein & & 11 & 1 & \\
\hline BHB & & 3 & 1 & \\
\hline Hospital Oberndorf & & 3 & 2 & \\
\hline Proctology & $n=52$ & 34 & 18 & $p=0.271$ \\
\hline PMU+Hallein & & 24 & 13 & \\
\hline BHB & & 10 & 5 & \\
\hline Hospital Oberndorf & & 0 & 0 & \\
\hline Revision operation & $\mathrm{n}=21$ & 17 & 4 & $p=0.421$ \\
\hline PMU+Hallein & & 15 & 4 & \\
\hline BHB & & 1 & 0 & \\
\hline Hospital Oberndorf & & 1 & 0 & \\
\hline Emergency thorax surgery & $n=29$ & 16 & 13 & $p=0.342$ \\
\hline PMU+Hallein & & 14 & 13 & \\
\hline BHB & & 2 & 0 & \\
\hline Hospital Oberndorf & & 0 & 0 & \\
\hline Abdominal trauma & $n=4$ & & & \\
\hline PMU+Hallein & & 2 & 2 & \\
\hline Stomach bleeding/perforation & $n=7$ & 3 & 4 & \\
\hline PMU+Hallein & & 3 & 4 & \\
\hline BHB & & 0 & 0 & \\
\hline Hospital Oberndorf & & 0 & 0 & \\
\hline
\end{tabular}




\begin{tabular}{|c|c|c|c|c|}
\hline & Period 1.3.-15.4.2019 & Period 1.3.-15.4.2020 & Total & \\
\hline Age & Median & Median & & \\
\hline Median & $52(+/-20,3)$ & $52(20,1)$ & & $p=0.991$ \\
\hline PMU+Hallein & $51(+/-20,5)$ & $54(+/-19,8)$ & & $p=0.867$ \\
\hline $\mathrm{BHB}$ & $54(+/-20,9)$ & $51(+/-19,2)$ & & $p=0.859$ \\
\hline Hospital Oberndorf & $51,5(+/-21,8)$ & $43(+/-19,0)$ & & $p=0.678$ \\
\hline Gender & Male(Female) & Male(Female) & Male(Female) & \\
\hline Total & $144(143)=287$ & $104(61)=165$ & $248(204)=452$ & $p=0.005$ \\
\hline PMU+Hallein & 104(99) & $71(30)$ & $175(129)=\mathbf{3 0 4}$ & \\
\hline $\mathrm{BHB}$ & $22(32)$ & $23(18)$ & $45(50)=95$ & \\
\hline Hospital Oberndorf & 18(12) & $10(13)$ & $28(25)=53$ & \\
\hline LOS (Lenght of Stay) & Median & Median & & \\
\hline Median & $4(0-79)$ & $3(0-49)$ & & \\
\hline PMU+Hallein & $3,5(+/-15,9)$ & $3,4(+/-8,1)$ & & $p=0.917$ \\
\hline $\mathrm{BHB}$ & $4(+/-5,7)$ & $3(+/-8,6)$ & & $p=0.873$ \\
\hline Hospital Oberndorf & $3(+/-15,3)$ & $5(+/-6,4)$ & & $p=0.724$ \\
\hline \multicolumn{5}{|l|}{ Mortality } \\
\hline PMU+Hallein & 12 & 1 & & \\
\hline BHB & 2 & 0 & & \\
\hline Hospital Oberndorf & 0 & 0 & & \\
\hline
\end{tabular}

\section{Figures}




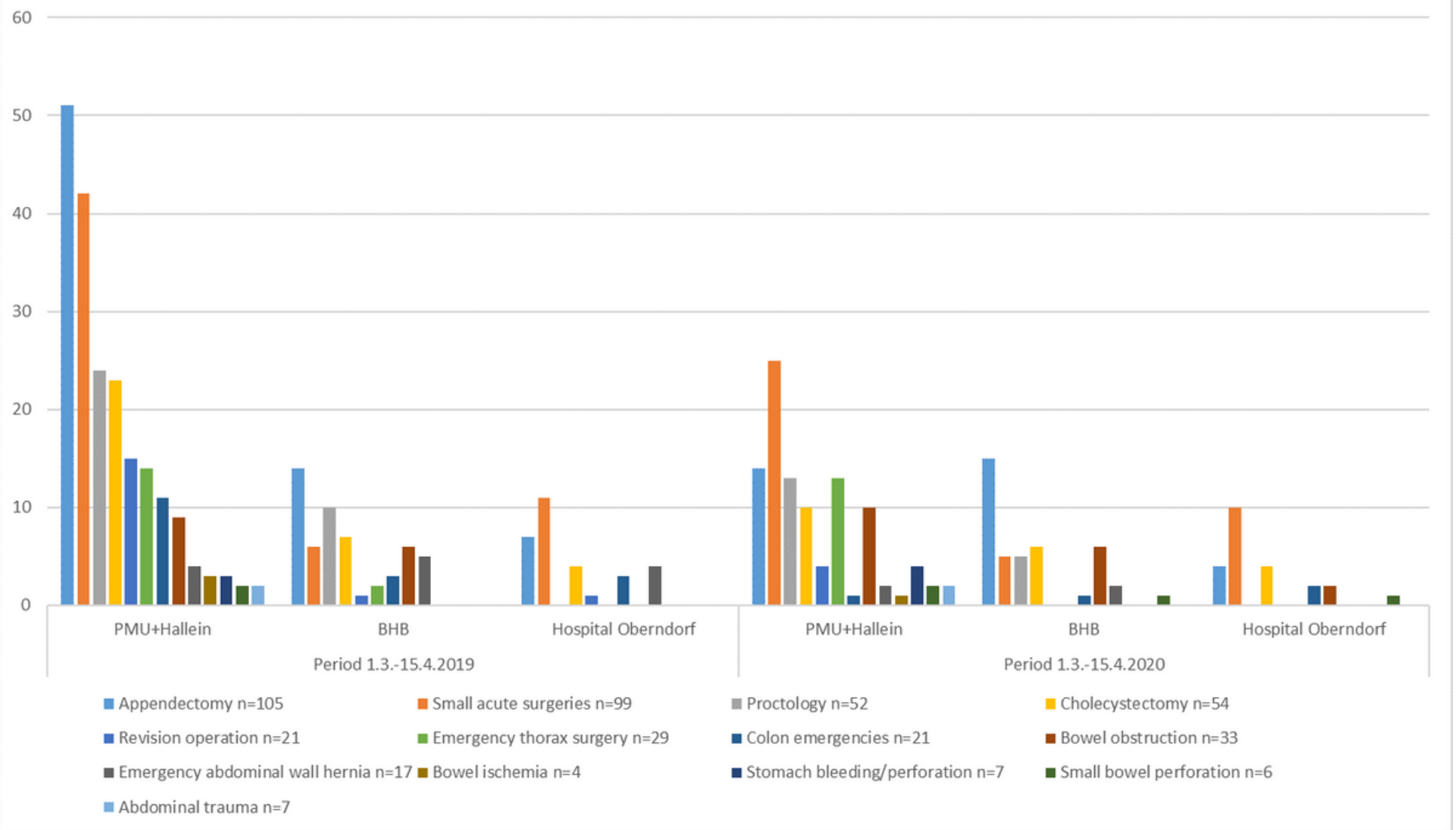

\section{Figure 1}

Figure 1 